\title{
Locomotor effects of d-amphetamine and methylphenidate in young squirrel monkeys
}

\author{
WALTER ISAAC and MARY D. KALLMAN \\ University of Georgia, Athens, Georgia 30602
}

\begin{abstract}
The effects of d-amphetamine and methylphenidate upon the locomotor activity of six young squirrel monkeys were studied. While d-amphetamine produced a dose-related decrease in activity, methylphenidate produced no significant changes. Combined doses of the drugs, as well as a large dose of methylphenidate, produced no changes in locomotor activity that were related to methylphenidate.
\end{abstract}

Considerable interest has been shown in the behavioral effects of two compounds, d-amphetamine sulfate (d-A), or Dexadrine, and methylphenidate hydrochloride (MP), or Ritalin. Both drugs are classified as stimulants (Goodman \& Gilman, 1965), yet they are used clinically in the treatment of hyperkinetic children, in whom they have been found to exert a "paradoxical" quieting effect (Fischer \& Wilson, 1971; Werry \& Sprague, 1970). Both drugs have been found to increase the level of locomotor activity of the rat, with the major difference observed being one of potency (Kallman \& Isaac, 1975). While both drugs did increase total activity, they had their greatest effect in the light.

In studies of the effect of d-A on the locomotor activity of monkeys, the drug was observed to have effects upon the nocturnal owl monkey similar to those observed in the rat (Isaac \& Troelstrup, 1969). However, in the diurnal monkeys, it has been found that d-A decreases locomotor activity in the rhesus macaque (Alexander \& Isaac, 1965) and the squirrel monkey (Isaac \& Troelstrup, 1969).

Our interest in comparing the effects of d-A and MP upon locomotor activity in the squirrel monkey arises from the similar interactions that they show with the level of ambient illumination during behavior measurement of the rat (Kallman \& Isaac, 1975). The interaction has been implicated in the discussion of the opposite effects of d-A upon locomotor activity levels of nocturnal and diurnal animals (Isaac \& Troelstrup, 1969).

\section{METHOD}

\section{Subjects}

Six squirrel monkeys, approximately 1 year old, were used as subjects. Sixty days of adaptation to the laboratory monkey colony preceded this investigation. All monkeys were housed in pairs in the colony room. Colony room illumination was maintained on a 12-h light, 12-h dark schedule. Daily diet consisted of Purina monkey chow, a vitamin supplement on $1 / 4$ slice of

This work was supported by a research grant (MH-25165) to the first author from the National Institute of Mental Health. M. D. Kallman's present address is the Department of Pharmacology, Medical College of Virginia, Richmond, Virginia. wheat bread, fresh fruit, and lettuce, with free access to water.

\section{Apparatus}

Expanded metal carrying cages $38 \mathrm{~cm}$ long, $24 \mathrm{~cm}$ wide, and $23 \mathrm{~cm}$ high served as activity chambers. Each carrying cage was centered in a sound-treated cubicle $56 \mathrm{~cm}$ long, $49 \mathrm{~cm}$ wide, and $83 \mathrm{~cm}$ deep in a sound-attenuated room. Approximately $50 \mathrm{fc}$ indirect illumination was provided to the floor of the carrying cage by a $20-\mathrm{W}$ shielded flourescent bulb mounted on the ceiling of each cubicle. Employing a method of sampling locomotor activity that has been found to be correlated with several other such measures (Isaac \& Ruch, 1956) and has also been found to be correlated with an observer evaluation of drug effects (Lowther \& Isaac, 1976), a single infrared light beam bisected the length of the carrying cage. When the beam of light, which was focused on a photocell, was interrupted by the movement of the monkey, a count was recorded on an electromechanical counter housed in a separate room.

\section{Procedure}

Each subject was adapted to the experimenter, the activity room, and the activity chamber for 9 months. During adaptation each monkey was trained to leave the home cage and enter a carrying cage where sweetened Hawaiian Punch was administered through a medicine dropper. After the monkey received the punch, it was carried out of the colony room and placed in an activity cubicle in another room. Following the initial exposures to the activity room, each monkey remained in the cubicle for $65 \mathrm{~min}$, with the first $5 \mathrm{~min}$ serving as a daily adaptation period.

After adaptation, drug administration began. Half of the subjects were assigned to d-A administration, and the other half were given MP. ${ }^{1}$ Six dose levels were examined for each drug: d-A-placebo, .05, .10, .20, $40, .80 \mathrm{mg} / \mathrm{kg}$; MP-placebo, $.10, .20, .40, .80,1.6 \mathrm{mg} / \mathrm{kg}$. Each subject received a counterbalanced sequence of daily doses assigned from a latin square. Each day all animals received $1 / 4$ slice of bread with a vitamin supplement approximately $4 \mathrm{~h}$ before testing; other food was withheld until testing was completed. To make oral administration of the drugs more palatable, the drugs were diluted in $8 \mathrm{cc}$ of sweetened Hawaiian Punch. Approximately $20 \mathrm{~min}$ elapsed from the time of drug administration until activity was sampled. Subject weights for determining drug doses on a milligram per kilogram basis were recalculated every 6 days.

Following completion of four replications ( 24 days) on one drug, the monkeys were given 2 days off drug and then switched to the other drug condition for four replications (24 days). Only the last two replications were analyzed, since several days were required for the adaptation of the monkeys to the altered taste of the fruit punch produced by the addition of the drug. This procedure also allowed the subjects to adapt 
to any alteration in the internal stimulus condition produced by drug-related physiological changes. By the beginning of the last two replications, all monkeys were taking the drugged punch readily and reliably. After activity had been sampled for four replications under both drug conditions, the animals were given 2 days off drug, followed by 5 consecutive days on a combined drug dose of $.2 \mathrm{mg} / \mathrm{kg} \mathrm{d}-\mathrm{A}$ and $.4 \mathrm{mg} / \mathrm{kg} \mathrm{MP}$, then 2 days off drug, followed by 5 consecutive days on $3.2 \mathrm{mg} / \mathrm{kg}$ MP.

\section{RESULTS}

A square-root transformation was performed on the scores, as recommended for frequency data by Edwards (1960). The scores from the last two replications under each drug were combined, after the transformation, to provide a more stable measure for each animal. The results are shown in Figure 1.

The effects of d-A upon locomotor activity in the squirrel monkey are similar to those observed previously (Isaac \& Troelstrup, 1969). Relatively small doses produced decreases in activity $[F(5,20)=5.11, p<.01]$. $\mathrm{MP}$, however, did not produce any measurable changes in activity $[\mathrm{F}(5,20)=1.71, \mathrm{p}>.10]$.

When the activity scores from the two replications included in the analysis were compared, no significant differences were observed under either d-A $[F(1,4)<1]$ or MP $[F(1,4)<1]$. Further, in comparison of the effects of the drugs related to whether they were the first drug given or the second drug given, the order effect of neither d-A $[F(1,4)<1]$ nor $\operatorname{MP}[F(1,4)<1]$ was significant.

A combined dose of $.2 \mathrm{mg} / \mathrm{kg} \mathrm{d}-\mathrm{A}$ and $.4 \mathrm{mg} / \mathrm{kg} \mathrm{MP}$ given for 5 consecutive days produced no change in activity over the period $[F(4,16)=1.03, p>.10]$. Further, the mean level of activity over the 5-day period did not differ from the mean level of activity observed under the placebo condition of the earlier phase of the study $[F(1,4)=2.82, p>.10]$.

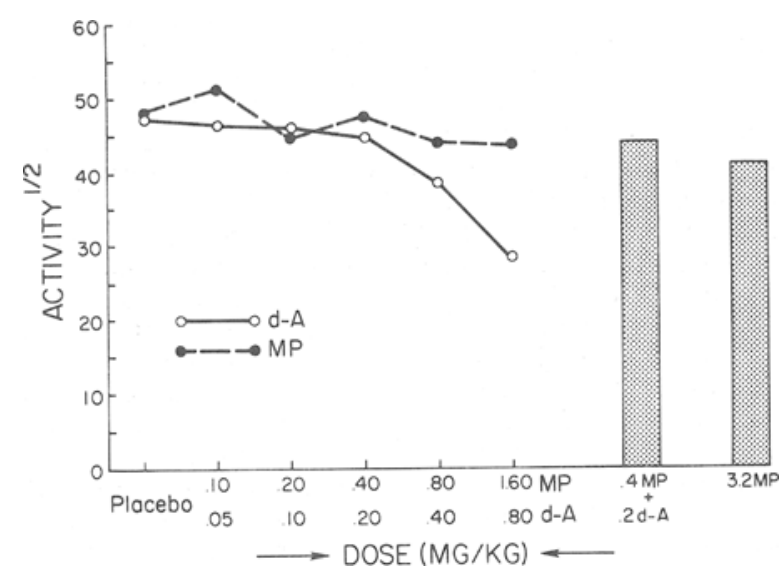

Figure 1. Mean level of activity (square-root transformed) with oral doses of d-amphetamine (d-A) and methylphenidate (MP) singly and in combination.
In the last portion of the study, it was found that a dose of $3.2 \mathrm{mg} / \mathrm{kg}$ of MP administered for 5 consecutive days produced no measurable changes in activity over the period $[F(4,16)<1]$. Again, the mean level of activity did not differ from the placebo condition of the earlier phase $[F(1,4)<1]$. At no time were stereotyped behaviors seen in the monkeys, such as we have observed with the next higher dose in the progression of $d$-A doses.

\section{DISCUSSION}

It is difficult to distinguish between behavioral changes associated with habituation to an altered stimulus condition produced by the effects of a drug and tolerance to the effects of that drug, since even a placebo may initially produce behavior changes (Isaac \& Isaac, 1977). However, the major change we have observed with continuing measurement of the effects of these drugs over several replications under the conditions described in the present study is an increased stability in the response to the drugs. The lack of a significant difference between the two replications under each drug that was used in the analysis indicates that the monkeys had habituated to the experimental conditions and the behavior had stabilized.

It is apparent that the effects of d-A and MP upon locomotor activity are not equivalent. While both drugs are classified as stimulants and have similar effects upon locomotor activity of the rat (Kallman \& Isaac, 1975), d-A serves to reduce the locomotor activity of the squirrel monkey, and MP has no observable effect. On the basis of earlier work (Lowther \& Isaac, 1976) and preliminary observations, the absence of stereotyped behavior at the oral dose levels was to be expected.

Since both of the drugs under investigation are important for their therapeutic value, an attempt has been made to study dose levels of the drugs that might be therapeutically meaningful. In an attempt to establish an equivalent dose for the squirrel monkey to the therapeutic dose in man, several sources were surveyed. The doses recommended would indicate that $15 \mathrm{mg}$ of both drugs would be reasonable. Transforming this dose to one that is equivalent in the squirrel monkey would indicate that a dose of approximately $.1 \mathrm{mg}$ is equivalent, if body weight is used to calculate the dosage, while a dose of approximately $.3 \mathrm{mg}$ is equivalent if body surface area is used to calculate the dosage. The dose range employed in the present study encompassed both values.

The finding that a dose equivalent to the therapeutic dose of d-A produced a behavioral change while a comparable dose of MP did not was surprising. The lack of a potentiation of the effect produced by the drugs when they were combined at medium dose levels and the lack of an effect that was seen with the largest dose of MP when it was given over a period of several days were also surprising. These data, however, are consistent with the recent finding of Goethe and Isaac (1977), who studied the effects of these drugs on a fixed-interval operant task.

Objective data concerning the effects of MP on motor behavior of the normal human subject is lacking. It has been proposed that MP has its "paradoxical" effect only in combination with damage to the central nervous system (Lasagna \& Epstein, 1970). Another possibility is that MP, because of a metabolic or absorption anomaly, is not effective in altering neural mechanisms related to the locomotor activity of the squirrel monkey. Examples of such unusual responses have been seen in other situations, such as the abnormal caffeine excretion pattern seen in the squirrel monkey (Burg, Burrows, \& Kensler, 1973). Until more information is available concerning the mechanism of action of this drug, however, such explanations of the present data are not possible. 


\section{REFERENCES}

Alexander, M., \& Isaac, W. Effects of illumination and d-amphetamine on the activity of the rhesus macaque. Psychological Reports, 1965, 16, 311-315.

Burg, A. W., Burrows, R., \& Kensler, C. J. Unusual metabolism of caffeine in the squirrel monkey. Toxicology and Applied Pharmacology, 1973, 28, 162-166.

EDWARDS, A. L. Experimental design in psychological research. New York: Rinehart, 1960.

Fischer, K., \& Wilson, W. Methylphenidate and the hyperkinetic state. Diseases of the Nervous System, 1971, 32, 695-698.

Goethe, K. E., \& IsAac, W. The effects of amphetamine and methylphenidate on fixed-interval responding in the squirrel monkey. Pharmacology, Biochemistry \& Behavior, 1977, 7, 78-83.

Goodman, L. S., \& Gilman, A. The pharmacological basis of therapeutics. New York: Macmillan, 1965.

IsAac, W. L., \& IsAAC, W. Differences in placebo effects. Pharmacology, Biochemistry \& Behavior, 1977, 6, 235-236.

IsaAC, W., \& Ruch, T. C. Evaluation of four activity techniques in monkeys. Science, 1956, 123, 1170.

IsaAc, W., \& Troelstrup, R. Opposite effects of illumination and d-amphetamine upon activity in the squirrel monkey
(Saimiri) and owl monkey (Aotes). Psychopharmacologia (Berlin), 1969, 15, 260-264.

Kallman, W. M., \& IsaAC, W. The effects of age and illumination on the dose-response curves for three stimulants. Psychopharmacologia (Berlin), 1975, 40, 313-318.

LASAgna, L., \& Epstein, L. C. The use of amphetamines in the treatment of hyperkinetic children. In E. Costa \& S. Garattini (Eds.), International symposium on amphetamine and related compounds. New York: Raven Press, 1970.

Lowther, W. R., \& IsAac, W. The effects of d-amphetamine and illumination on behaviors of the squirrel monkey. Psychopharmacology, 1976, 50, 231-235.

Werry, J., \& Sprague, R. Hyperactivity. In C. Costello (Ed.), Symptoms of psychopathology. New York: Wiley, 1970.

\section{NOTE}

1. The d-amphetamine sulfate was generously provided by Smith, Kline, and French Laboratories; the methylphenidate hydrochloride was generously provided by the Ciba-Geigy Corporation.

(Received for publication July $25,1979$. ) 